\title{
Article \\ The Impact of Neurocognitive Functioning on the Course of Posttraumatic Stress Symptoms following Civilian Traumatic Brain Injury
}

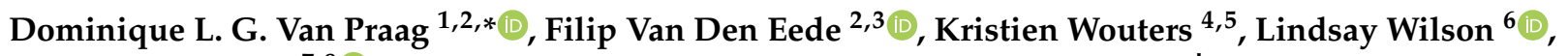 \\ Andrew I. R. Maas $7,8(1)$ and The CENTER-TBI Investigators and Participants ${ }^{\dagger}$ \\ 1 Department of Psychology, Antwerp University Hospital, 2650 Edegem, Belgium \\ 2 Collaborative Antwerp Psychiatric Research Institute, University of Antwerp, 2610 Wilrijk, Belgium \\ 3 Department of Psychiatry, Antwerp University Hospital, 2650 Edegem, Belgium; filip.vandeneede@uza.be \\ 4 Clinical Trial Center, Clinical Research Center Antwerp, Antwerp University Hospital, \\ 2650 Edegem, Belgium; kristien.wouters@uza.be \\ 5 Antwerp Research Center, Faculty of Medicine, University of Antwerp, 2610 Wilrijk, Belgium \\ 6 Division of Psychology, University of Stirling, Stirling FK9 4LA, UK; l.wilson@stir.ac.uk \\ 7 Department of Neurosurgery, Antwerp University Hospital, 2650 Edegem, Belgium; andrew.maas@uza.be \\ 8 Translational Neurosciences, University of Antwerp, 2610 Wilrijk, Belgium \\ Correspondence: dominique.vanpraag@uza.be; Tel.: +32-3-821-56-14 \\ $+\quad$ The CENTER-TBI Participants and Investigators Are Provided in the Supplementary Materials.
}

Citation: Van Praag, D.L.G.; Van Den Eede, F.; Wouters, K.; Wilson, L.; Maas, A.I.R.; The CENTER-TBI Investigators and Participants. The Impact of Neurocognitive

Functioning on the Course of Posttraumatic Stress Symptoms following Civilian Traumatic Brain Injury. J. Clin. Med. 2021, 10, 5109. https://doi.org/10.3390/jcm10215109

Academic Editors: Elena Tomba and Allan H. Young

Received: 1 August 2021

Accepted: 28 October 2021

Published: 30 October 2021

Publisher's Note: MDPI stays neutral with regard to jurisdictional claims in published maps and institutional affiliations.

Copyright: (c) 2021 by the authors. Licensee MDPI, Basel, Switzerland. This article is an open access article distributed under the terms and conditions of the Creative Commons Attribution (CC BY) license (https:/ / creativecommons.org/licenses/by/ $4.0 /)$.

Abstract: Background: One out of seven individuals who have suffered a traumatic brain injury (TBI) develops a posttraumatic stress disorder (PTSD), which is often associated with neurocognitive impairment. The present study explores the impact of neurocognitive functioning after mild, moderate, and severe TBI on the course of PTSD symptoms. Methods: The data of 671 adults admitted to hospital for a TBI was drawn from the Collaborative European Neurotrauma Effectiveness Research (CENTER-TBI) study. After six- and 12-months post-injury, participants completed the PTSD Checklist-5 (PCL-5), from which change scores were calculated. At six months, participants also completed a neurocognitive assessment including the Rey Auditory Verbal Learning Test, the Trail Making Test, and the Cambridge Neuropsychological Test Automated Battery (CANTAB). Linear regressions were performed to identify associations between cognitive functioning and PCL- 5 change scores. Results: Overall, mean PCL-5 change scores showed no clear change $(-0.20 \pm 9.88)$, but 87 improved and 80 deteriorated by a change score of 10 or more. CANTAB Rapid Visual Information Processing scores were significantly associated with PCL-5 change scores. Conclusions: Strong sustained attention was associated with improvement in PTSD symptoms. Assessing cognitive performance may help identify individuals at risk of developing (persisting) PTSD post-TBI and offer opportunities for informing treatment strategies.

Keywords: assessment; cognition; neuropsychology; posttraumatic stress; concussion; head injury

\section{Introduction}

Considering that $15 \%$ of patients suffering from mild traumatic brain injury (TBI), and $65 \%$ suffering from moderate or severe TBI, reported long-lasting cognitive problems and functional disability [1,2], it is evident that TBI is a chronic and incapacitating condition for many people. In addition to the cognitive, emotional, and physical consequences [3], in the case of civilian TBI, posttraumatic stress disorder (PTSD) is diagnosed in 14\% [4,5]. TBI is a clear risk factor for PTSD, possibly through underlying neurobiological damage and impaired cognitive resources [6,7]. Poor cognitive performance may stem from the TBI but also, or alternatively, from post-TBI PTSD, since there is an overlap in the cognitive symptoms resulting from the two conditions, such as impaired speed of information processing, attention, working memory, learning and verbal memory [8-10]. Nonetheless, 
studies have reported differences in the cognitive deficits of patients with and without PTSD after mild TBI [11,12]. In a previous study by our research group, low cognitive flexibility and impaired long-term verbal memory were more strongly associated with probable PTSD than with TBI only [13]. Establishing differential cognitive profiles for cooccurring PTSD and TBI is crucial to informing treatment that targets cognitive impairment. Importantly, cognitive training after TBI should not merely focus on documented cognitive weaknesses but also on boosting (the remaining) protective cognitive resources. Activating the neurocognitive reserve may potentially protect against the development of PTSD in the longer term or help to reduce PTSD symptoms by enhancing the control over trauma memories and emotions while inhibiting maladaptive thoughts and producing constructive alternatives [14,15].

Vasterling et al. (2018) found that impairment of visual and verbal memory was related to an increase in PTSD severity over time [16]. They suggest that strong visual and verbal memory might help TBI survivors reconstruct their trauma narratives better and thus facilitate their processing of the traumatic event [17]. In contrast, Guo et al. (2017) report that in children, deficits in working memory and verbal learning three months postTBI protected against PTSD at six months, while poor sustained attention predicted PTSD symptoms [18]. However, since these studies were conducted in military and paediatric populations, the results may not be generalizable to the broader context of adults having sustained TBIs in a civilian setting $[16,18]$. Additionally, the two studies covered either a relatively short or a long time span (up to 6 months versus an average of 7 years). As longitudinal research shows a peak in the prevalence of PTSD between six and 12 months after moderate to severe TBI, we considered it relevant to investigate symptom change within this timeframe [19].

The present longitudinal study builds on our previous work, in which we found low cognitive flexibility and impaired long-term verbal memory to be associated with probable PTSD in adults having suffered a civilian TBI [13]. Here, our primary aim is to describe the course of PTSD symptoms, more specifically to clarify the impact of neurocognitive functioning six months post-TBI on the course of PTSD symptoms between six and 12 months, where we hypothesise that strong visual and verbal memory will coincide with a reduction in PTSD symptoms. Our second aim is to identify the cognitive capacities that are key to an overall change in PTSD symptoms for each of the four PTSD clusters (i.e., intrusion, avoidance, cognition and mood, and arousal), which, to our knowledge, has not been previously studied in detail. In general, we expect that improved cognitive performance will be related to a decrease in PTSD symptoms $[9,18,20]$. We further anticipate that our exploratory analyses at the PTSD-cluster level will provide us with new insight into the relationship between cognition and PTSD-specific (intrusion) and non-specific symptoms (cognition and mood, and arousal). In addition to our main objectives, we will explore the evolution of PTSD by looking at the prevalence and recovery of 'early' PTSD at six months and the onset of 'delayed' PTSD 12 months post trauma.

\section{Materials and Methods}

\subsection{Study Participants and Procedure}

Participants with a clinical diagnosis of TBI were drawn from the European CENTERTBI Core study (Collaborative European Neurotrauma Effectiveness Research: www.centertbi.eu (accessed on 30 July 2021)), a prospective, observational study with 65 recruiting sites in 19 countries. Aimed at characterising TBI more closely and finding the most effective TBI treatment approaches (clinicaltrials.gov NCT02210221) [21], only participants who had presented at a study site within $24 \mathrm{~h}$ of the injury and who had had a clinical indication for CT-scan were included [22]. Pre-existing severe neurological disorders that could confound outcome assessments was an exclusion criterion. Participants were recruited in three strata: discharge from the accident and emergency room, admission to a hospital ward, or admission to the ICU. For the current study, we selected patients from the latter two strata only because, according to protocol, they were scheduled for the 12-month follow-up. 
Furthermore, participants needed to be over 16 years of age and have a 6-month Glasgow Outcome Score Extended (GOSE) [23] above 3, to thus exclude individuals who were likely to be unable to complete the cognitive assessment.

Research personnel collected demographic, pre-injury history, and injury-related information at recruitment. At the 6- and 12-month follow-up visits, self-report questionnaires were administered; at the 6-month follow-up, a trained research nurse or neuropsychologist administered the cognitive test battery. Research personnel were instructed to record test validity issues using test completion codes [24], and results flagged as invalid were removed. All data were entered into an electronic case report form, de-identified and stored in a secure database. Central or local Institutional Review Boards approved the protocol for all recruiting sites, and informed consent was obtained from all participants or their legally acceptable representative in accordance with national and local regulations. More information can be found on: https:/ / www.center-tbi.eu/project/ethical-approval (accessed on 30 July 2021).

\subsection{Measures}

The Posttraumatic Stress Disorder Checklist, DSM-5 (PCL-5), is a self-report questionnaire to screen for PTSD, measure symptom severity and monitor change over time [25]. Respondents are asked to rate its 20 items that are based on the DSM- 5 diagnostic criteria of PTSD on a scale ranging from 0 (not at all bothered) to 4 (extremely bothered), with a maximum total score of 80 . Change scores can be calculated by subtracting the PCL-5 total score at six months from the PCL- 5 total score at 12 months. Although change scores need to be determined and validated, a change of 10 points on the PCL- 5 is considered clinically relevant, similar to the threshold for the PCL for the DSM-IV [25,26]. A probable diagnosis of PTSD can be made using the symptom cluster method that considers the DSM- 5 cluster criteria $[25,27]$, with items $1-5$ reflecting intrusion symptoms, items 6 and 7 avoidance symptoms, items 8-14 cognition and mood, and items 15-20 arousal. A diagnosis requires at least a score of 2 or higher on one intrusion item, one avoidance item, two items on negative alterations in cognitions and mood and two items on alterations in arousal and reactivity. Cluster scores are calculated by adding these clinically relevant items. Below, we will alternately use the terms PTSD or probable or suspected PTSD when referring to the participants who met the PCL-5 criteria for PTSD. Note that to confirm the PCL-5 diagnosis, a thorough evaluation by a psychiatrist is required.

The Trail Making Test (TMT) is a pen-and-paper test assessing information processing, attention, and cognitive flexibility [28]. In Part A, the participant is instructed to connect numbers sequentially, as fast as possible, within $100 \mathrm{~s}$ and in Part B, numbers and letters alternately within $300 \mathrm{~s}$.

The Rey Auditory Verbal Learning Test (RAVLT) assesses verbal learning and memory, and consists of a list of 15 unrelated words that are read to the participant, who is instructed to try and remember, and verbally reproduce as many words as possible $[29,30]$. This is repeated five times (trials 1-5: immediate recall), after which a second, so-called interference list is read out, which the participant needs to repeat once. Immediately after reproducing the interference words, the participant is asked to recollect words from the main list (trial 6: interference recall) and to recall the words again after $20 \mathrm{~min}$ (trial 7: delayed recall). The sum of the first five trials and trial six are used as a measure of short-term memory and the last trial as a measure of long-term memory.

The Cambridge Neuropsychological Test Automated Battery (CANTAB) is a computerised battery gauging diverse cognitive domains [31]. CANTAB mainly uses nonverbal stimuli in order to be language- and culture-independent. The reaction time task (RTI), the attention switching task (AST), the spatial working memory task (SWM), the paired associate learning task (PAL), the rapid visual information processing task (RVP) and the Stockings of Cambridge task (SOC) were selected for the present study. The RTI measures the intervals between the onset of the stimuli and the moments at which the participant releases the button, with the median duration serving as the outcome measure. The AST 
assesses cognitive flexibility by measuring the difference between response times for trial blocks in which the rule was switched and those for blocks in which the rule remained the same. Scores close to 0 indicate strong cognitive flexibility, since they reflect little variation in response times between switch and non-switch trials. The SWM assesses working memory by recording the number of errors made in finding hidden tokens relative to the maximum number of errors that could have been made. The PAL gauges visuospatial learning and memory. The number of errors is recorded and again compared to the maximum number of errors possible. Testing sustained attention and concentration, the RVP requires the participant to detect a specific sequence of digits; detection sensitivity is the outcome measure, with scores close to 1 reflecting strong sustained attention. Finally, the SOC tests for executive functioning, and more specifically for problem-solving strategies and spatial planning. The outcome is based on the number of trials completed in the smallest possible number of moves. See Table S1 for additional information on the TMT, RAVLT and CANTAB variables.

\subsection{Statistical Analysis}

SPSS version 25 was employed for all analyses [32]. CENTER-TBI data (version 2.1) were accessed using Neurobot, a bespoke data management tool (https: / neurobot.incf.org (accessed on 1 July 2021)).

The cohort sample was described using the median and interquartile range (IQR) for continuous variables and the frequency and percentages for categorical variables. The PCL5 change score was used to delineate the course of PTSD symptoms by subtracting the PCL5 sum score at 6 months from the PCL- 5 sum score at 12 months. In addition, we described the evolution of PTSD symptoms over time (from 6 to 12 months post trauma). Using the symptom cluster method, we identified four TBI groups: improved (suspected PTSD at 6 months only), delayed-onset PTSD (suspected PTSD at 12 months only), persistent PTSD (suspected PTSD both at 6 and 12 months) and resilient (no suspected PTSD at 6 or 12 months).

To determine the impact of neurocognitive functioning on the course of PTSD (cluster) symptoms, linear regressions were performed with the PCL-5 (cluster) change score as the dependent variable. Age, gender, educational level, psychiatric history, and the baseline Glasgow Coma Score (GCS) [33] were entered as demographic and injury-related covariates. The PCL-5 diagnosis at 6 months was entered to control for existing PTSD, as we expect the direction and sizes of change scores to be dependent on diagnostic status. The neurocognitive variables were TMT-A, TMT-B, RAVLT-Immediate recall, RAVLT-Interference recall, RAVLT-Delayed recall, CANTAB RTI, AST, PAL, SOC, SWM and RVP. All cognitive test scores were converted to z-scores for comparability across tests. In the case of nonnormality, variables were first log-transformed. We explored the interaction effects of TBI severity and cognitive test scores on the PCL- 5 change score. Model selection was based on covariate significance $(p<0.20)$ and adjusted $R^{2}$. Multicollinearity was checked by means of the variance inflation factor (VIF). Statistical significance was judged based on a more stringent $p$-value of $<0.01$ to decrease the risk of type-I errors.

\section{Results}

\subsection{Study Sample and Characteristics}

Of the 3661 patients of the CENTER-TBI Core study enrolled in the admission and ICU strata, 2072 met the inclusion criteria for this study. Of these, 671 participants had complete PCL-5 (6 + 12 months) and 6-month cognitive datasets and were included in the analyses (Figure 1). 


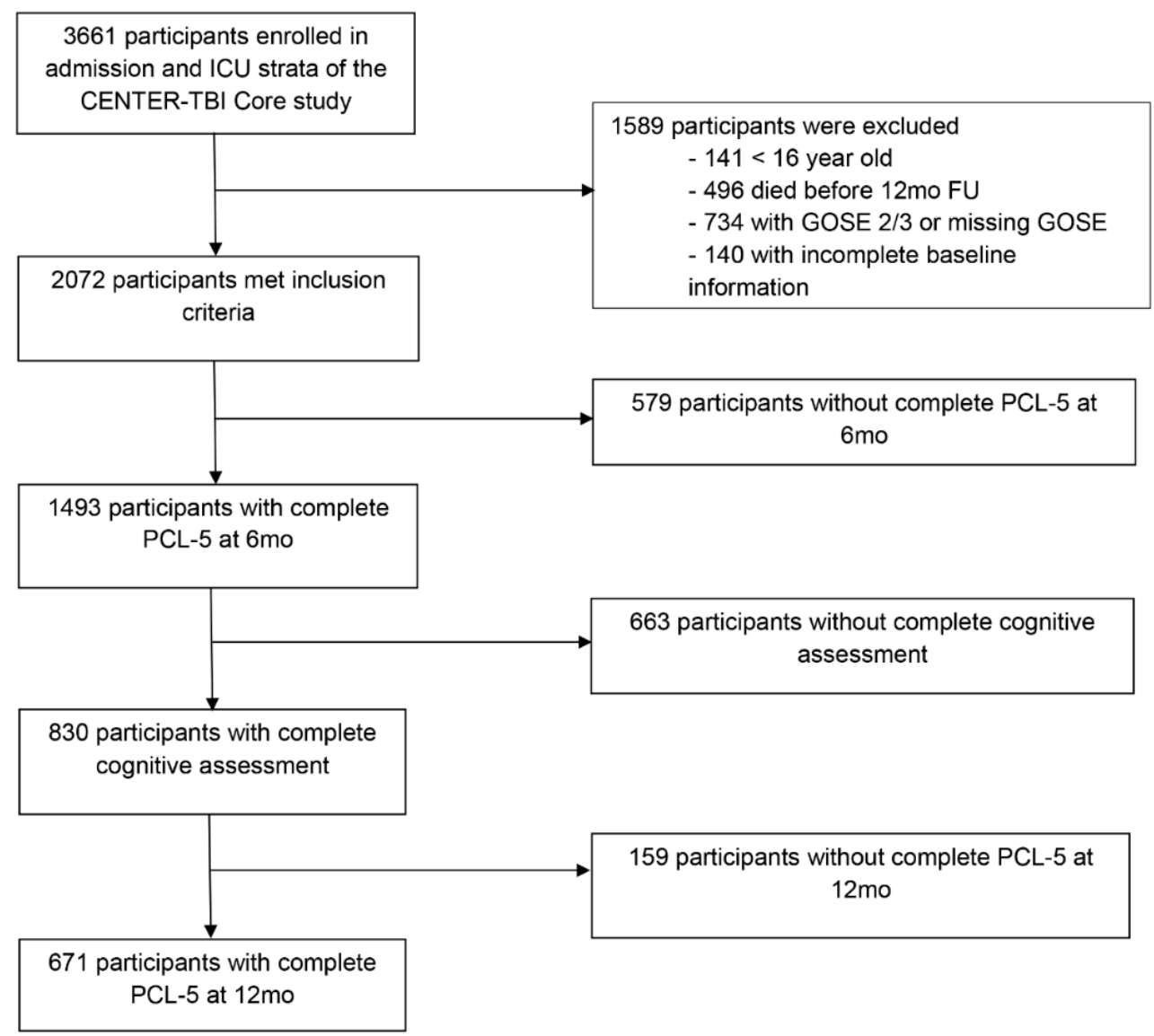

Figure 1. Participant inclusion flowchart.

Table 1 summarises the demographic and clinical characteristics of the study sample. The median age was 49 , two thirds were male, and $70.6 \%$ was classified at baseline as having mild, $10 \%$ moderate and $19.4 \%$ severe TBI. One in ten had a psychiatric history, which mostly concerned a depressive disorder. At six months, 79 participants $(11.8 \%)$ screened positive for PTSD and at 12 months, 71 participants screened positive $(10.6 \%)$. In Table S2, we list the characteristics of our sample and those of the participants $(n=1401)$ that met the inclusion criteria but had incomplete follow-up data. The participants lost to follow-up were older, had received less education and were more often single, living alone, or a combination thereof. In most participants of the study sample, TBI was sustained in traffic incidents, while in the lost-to-follow-up group, incidental falls were more frequent, with fewer suicide attempts or violent incidents being reported. Finally, in the lost-to-FU group, more people reported a history of substance abuse.

\subsection{Course of PTSD Symptoms}

The mean PCL-5 change score was -0.20 , with a standard deviation of 9.88 . A total of eighty-seven participants had improved (change score $\leq-10$ ) and 80 had deteriorated (change score $\geq 10$ ). The mean PCL-5 change score was significantly different (Hedges' $\mathrm{d}=1.03$ ) for the participants meeting the criteria for probable PTSD at six months $(\mathrm{M}=-8.75, \mathrm{SD}=14.06)$ than it was for those that $\operatorname{did} \operatorname{not}(\mathrm{M}=0.94, \mathrm{SD}=8.57)$, $\mathrm{t}(85.89)=5.98, p<0.001$, Figure 2$)$. Where the PTSD group showed a negative mean PCL-5 change score, reflecting an overall improvement of PTSD symptoms over time, the TBI-only group showed no overall statistically significant change. See Table S3 for the mean PCL-5 scores at 6 and 12 months. The mean PCL-5 cluster change scores are shown in Table S4 for the participants with and without probable PTSD. 
Table 1. Participant characteristics $(n=671)$.

\begin{tabular}{|c|c|}
\hline Demographic Characteristics & Median (IQR) ${ }^{1}$ or $n(\%)$ \\
\hline Age (years) & $49(31-61)$ \\
\hline$>65$ years & $102 / 671(15.2)$ \\
\hline Male & $463 / 671(69.0)$ \\
\hline \multicolumn{2}{|l|}{ Highest educational level: } \\
\hline College/University & $188 / 614(30.6)$ \\
\hline Married or living with partner & $369 / 648(56.9)$ \\
\hline \multicolumn{2}{|l|}{ Injury-related characteristics } \\
\hline \multicolumn{2}{|l|}{ Glasgow Coma Scale: } \\
\hline Mild TBI (13-15) & $474 / 671(70.6)$ \\
\hline Moderate TBI (9-12) & $67 / 671(10.0)$ \\
\hline Severe TBI (3-8) & $130 / 671(19.4)$ \\
\hline \multicolumn{2}{|l|}{ Cause of injury: } \\
\hline Road traffic incident & $321 / 656(48.9)$ \\
\hline Incidental fall & $244 / 656(37.2)$ \\
\hline Violence/assault/act of mass violence & $24 / 656(3.7)$ \\
\hline Suicide attempt & $12 / 656(1.8)$ \\
\hline \multicolumn{2}{|l|}{ Care pathway: } \\
\hline Admitted to hospital & $32 / 671(47.7)$ \\
\hline Intensive Care Unit & $351 / 671(52.3)$ \\
\hline \multicolumn{2}{|l|}{ Psychiatric history $^{2}$} \\
\hline Psychiatric disorders & $68 / 671(10.1)$ \\
\hline \multicolumn{2}{|l|}{ Type of psychiatric disorder: } \\
\hline Depression & $37 / 68(54.4)$ \\
\hline Anxiety & $18 / 68(26.5)$ \\
\hline Sleep disorder & $11 / 68(16.2)$ \\
\hline Substance abuse & $8 / 68(11.8)$ \\
\hline \multicolumn{2}{|l|}{ Psychiatric characteristics at follow-up } \\
\hline PCL $-5^{3}$ total score 6 months & $8(3-18)$ \\
\hline PTSD probable diagnosis 6 months & $79 / 671(11.8)$ \\
\hline PCL-5 total score 12 months & $8(2-18)$ \\
\hline PTSD probable diagnosis 12 months & $71 / 671(10.6)$ \\
\hline PHQ-9 ${ }^{4}$ total score 6 months & $3(1-7)$ \\
\hline GAD $-7^{5}$ total score 6 months & $2(0-5)$ \\
\hline Psychotropic medication at 6 months & $143 / 633(22.6)$ \\
\hline \multicolumn{2}{|l|}{ Type of medication: } \\
\hline Antidepressants & $38 / 143(26.6)$ \\
\hline Anxiolytics & $21 / 143(14.7)$ \\
\hline Antipsychotic agents & $11 / 143(7.7)$ \\
\hline
\end{tabular}

$\overline{{ }^{1} \mathrm{IQR}}=$ Interquartile range. ${ }^{2}$ Information on psychiatric history and type(s) of psychiatric disorder was obtained during the interview with the patient and/or caretaker upon hospital admission. ${ }^{3}$ PCL-5 is Posttraumatic Stress Disorder Checklist-5, ${ }^{4}$ The PHQ-9 is the Patient Health Questionnaire-9, a self-report questionnaire that screens for depressive symptoms. It includes 9 items and results in a maximum total score of 27 with high scores reflecting more severe symptoms, ${ }^{5}$ GAD-7 is Generalized Anxiety Disorder-7, a self-report questionnaire that screens for anxiety symptoms. It includes 7 items with a maximum total score of 21 , with high scores reflecting more severe symptoms.

The analysis investigating the evolution of PTSD symptoms over time revealed that of the 79 (of 671) participants with probable PTSD six months post-injury (11.8\%), 40 $(50.6 \%)$ had improved at 12 months. However, with 71 participants reporting symptoms at 12 months, PTSD prevalence had not significantly decreased relative to the 6-month assessment. In 39 of these 71 participants PTSD was persistent, while 32 had developed delayed-onset PTSD. The remaining 560 participants $(83.5 \%)$ had no PTSD diagnosis at either timepoint (resilient group). Figure 3 shows the relationships between the PCL5 scores at six months and the PCL-5 change scores differentiated for the four groups. 
Note that the maximum negative change score is dependent on the PCL-5 total score at six months, which, as can be seen in Figure 3, has a strong floor effect. In addition, Figure 3 shows regression to the mean of the PCL- 5 scores. More than $80 \%$ of the resilient participants showed no clinically relevant change in PCL-5 scores, but in half of those with a persistent PTSD, PCL-5 scores had improved or worsened (>10 points, 1 SD). Conversely, those having improved or those with delayed-onset PTSD, showed no significant changes in PCL-5 scores (resp. 20 and 30\%) and scored just above or close to the threshold at six months.

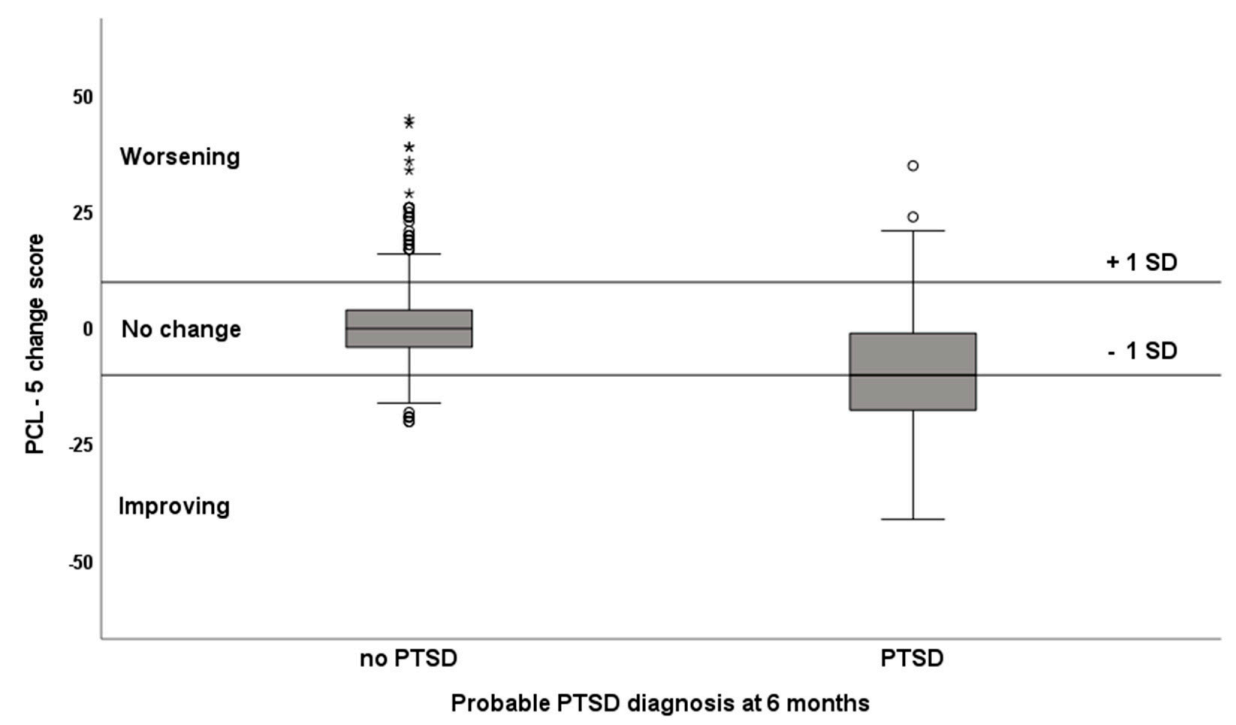

Figure 2. Boxplots of PCL-5 change scores differentiated for probable PTSD 6 months post-injury. The boxplots demonstrate the interquartile range and median of the PCL- 5 change scores for participants with and without probable PTSD at 6 months. The whiskers represent $1.5 \times$ IQ range, ${ }^{\circ}$ are outliers between 1.5 and $3 \times$ IQ range, * are outliers over $3 \times$ IQ range.

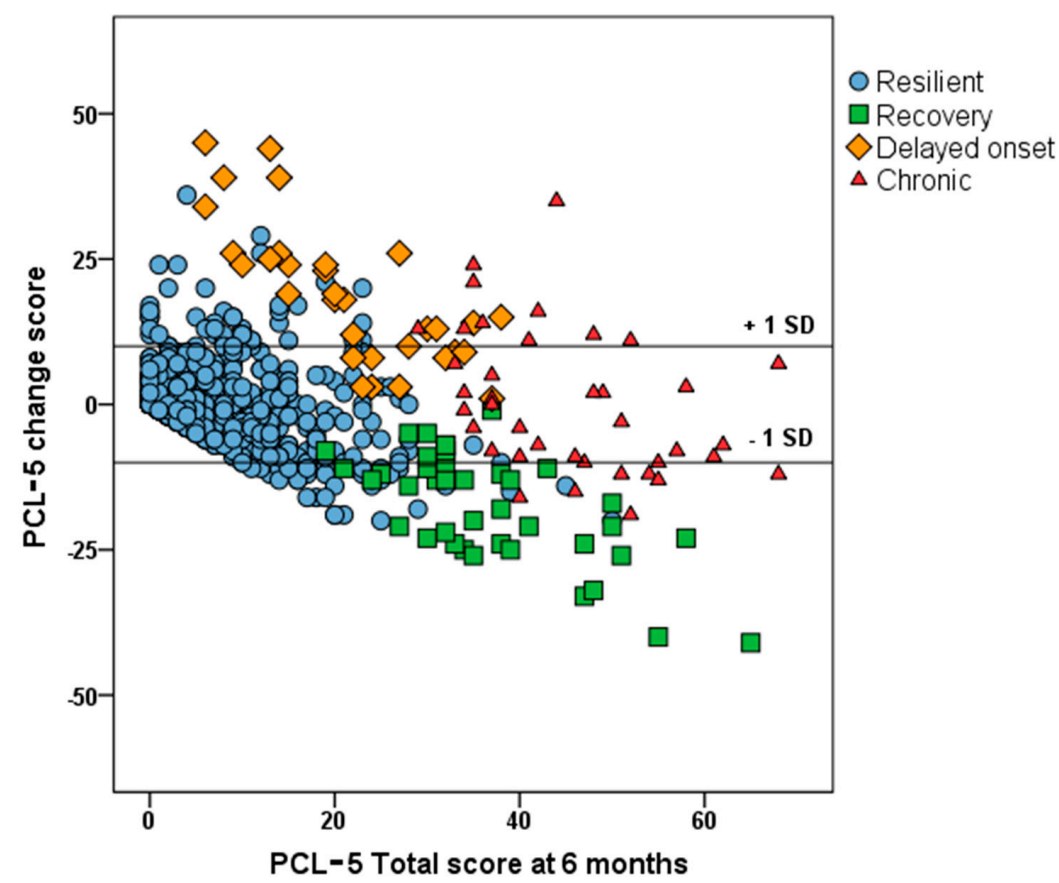

Figure 3. Scatterplot of PCL-5 change scores and PCL-5 total scores 6 months post-injury differentiated for PTSD at 6 and 12 months (resilient (83.5\%), improved (5.9\%), persistent (5.8\%) and delayed onset $(4.8 \%)$ ). 


\subsection{Cognitive Functioning and the Course of PTSD Symptoms}

A multiple linear regression model was calculated to associate PCL- 5 change scores with the cognitive test results (see Table 2, adjusted $R^{2}=0.14$ ). Age, psychiatric history, baseline GCS, probable PTSD diagnosis at six months, CANTAB RVP and AST were included in the final model. GCS was included as a continuous (ordinal) variable ranging from 3 to 15. Adding gender, educational level or other cognitive test outcomes had no added explanatory value. Baseline GCS was inversely related to changes in PTSD symptoms, with low GCS scores reflecting a severe injury and high PCL-5 change scores a worsening of PTSD symptoms (from 6 to 12 months). Suspicion of PTSD at six months was related to a reduction in PTSD symptoms, probably because of the room for improvement. Age and prior psychiatric illness showed no significant associations with PCL-5 change scores.

Table 2. Linear regression: covariates associated with PCL-5 change scores.

\begin{tabular}{|c|c|c|c|c|c|c|}
\hline \multirow[t]{2}{*}{ Covariate } & \multirow[t]{2}{*}{ B } & \multirow[t]{2}{*}{ SE (B) } & \multicolumn{2}{|c|}{$95 \% \mathrm{CI}^{1}$} & \multirow[t]{2}{*}{$p^{4}$} & \multirow[t]{2}{*}{ VIF $^{5}$} \\
\hline & & & $\mathrm{LL}^{2}$ & $\mathrm{UL}^{3}$ & & \\
\hline (Intercept) & -2.45 & 1.94 & -6.25 & 1.35 & 0.21 & \\
\hline Age & -0.032 & 0.022 & -0.075 & 0.012 & 0.15 & 1.22 \\
\hline Psychiatric history & 2.14 & 1.18 & -0.18 & 4.46 & 0.071 & 1.01 \\
\hline $\mathrm{GCS}^{6}$ & -0.27 & 0.095 & -0.46 & -0.082 & 0.005 & 1.23 \\
\hline PTSD diagnosis $6 \mathrm{mo}$ & -10.09 & 1.11 & -12.28 & -7.91 & $<0.001$ & 1.02 \\
\hline CANTAB $^{7}$ RVP $^{8}$ & -1.04 & 0.40 & -1.82 & -0.26 & 0.009 & 1.16 \\
\hline CANTAB AST $^{9}$ & 2.67 & 1.08 & 0.55 & 4.79 & 0.014 & 1.19 \\
\hline GCS $\times$ CANTAB AST & -0.21 & 0.086 & -0.38 & -0.037 & 0.017 & \\
\hline
\end{tabular}

${ }^{1} \mathrm{CI}=$ confidence interval; ${ }^{2} \mathrm{LL}=$ lower limit $;{ }^{3} \mathrm{UL}=$ upper limit; ${ }^{4}$ Significance level $p<0.01 ;{ }^{5} \mathrm{VIF}=$ variance inflation factor $;{ }^{6} \mathrm{GCS}=$ Glasgow Coma Scale $;{ }^{7}$ CANTAB $=$ Cambridge Neuropsychological Test Automated Battery $;{ }^{8} \mathrm{RVP}=$ Rapid Visual Information Processing test (high scores reflect strong sustained ability); ${ }^{9}$ AST $=$ Attention Switching Task (high scores reflect low cognitive flexibility), high PCL-5 change scores reflect worsening symptoms. The $\mathrm{R}^{2}$ change score for the CANTAB RVP is 0.008 .

RVP outcomes were significantly inversely associated with PCL-5 change scores, with high RVP scores (strong sustained attention) relating to negative change scores, thus reflecting improved PTSD symptoms. The association between AST outcomes (where higher scores reflect poorer cognitive flexibility) and change scores was not significant $(p=0.014)$.

A subgroup analysis was performed for the 79 participants with probable PTSD at six months, to investigate the associations between PTSD symptom course and cognitive functioning. See Table S5 for the linear regression model (adjusted $R^{2}=0.18$ ). Higher CANTAB PAL scores (weak visual learning and memory) were significantly associated with higher change scores, reflecting a deterioration in PTSD symptoms. Low RAVLTInterference recall scores (weak short-term verbal memory), but only in individuals with moderate and severe TBI, were significantly associated with higher PCL-5 change scores, reflecting improved PTSD symptoms.

\subsection{Cognitive Functioning and the Course of PTSD Cluster Symptoms}

The final models for the PTSD-cluster change scores are shown in Table 3. The change score for the intrusion and cognition and mood clusters were not significantly related to performance on any of the cognitive tests. 
Table 3. Linear regression analyses: covariates associated with PCL-5 cluster change scores.

\begin{tabular}{|c|c|c|c|c|c|c|c|c|c|c|c|c|}
\hline \multirow{2}{*}{ Covariates } & \multicolumn{3}{|c|}{$\begin{array}{l}\text { Cluster Intrusion } \\
\text { (5 Items) }\end{array}$} & \multicolumn{3}{|c|}{$\begin{array}{l}\text { Cluster Avoidance } \\
\text { (2 Items) }\end{array}$} & \multicolumn{3}{|c|}{$\begin{array}{c}\text { Cluster Cognition/Mood } \\
\text { (7 Items) }\end{array}$} & \multicolumn{3}{|c|}{$\begin{array}{c}\text { Cluster Arousal } \\
\text { (6 Items) }\end{array}$} \\
\hline & B (SE) & $p^{1}$ & VIF $^{2}$ & B (SE) & $p^{1}$ & VIF $^{2}$ & B (SE) & $p^{1}$ & VIF $^{2}$ & B (SE) & $p^{1}$ & VIF $^{2}$ \\
\hline Intercept & $-0.98(0.58)$ & 0.092 & & $-0.79(0.26)$ & 0.003 & & $2.32(0.84)$ & 0.006 & & $-0.84(0.63)$ & 0.18 & \\
\hline Age & & & & & & & $-0.014(0.010)$ & 0.16 & 1.13 & & & \\
\hline Psychiatric history & $0.84(0.38)$ & 0.027 & 1.01 & & & & $0.25(0.57)$ & 0.66 & 1.00 & $0.53(0.41)$ & 0.20 & 1.01 \\
\hline $\mathrm{GCS}^{3}$ & $-0.051(0.029)$ & 0.080 & 1.02 & $-0.029(0.016)$ & 0.060 & 1.03 & $-0.12(0.046)$ & 0.007 & 1.11 & $-0.092(0.031)$ & 0.003 & 1.03 \\
\hline CANTAB $^{4}$ RVP $^{5}$ & $-0.23(0.12)$ & 0.053 & 1.03 & & & & $-0.40(0.19)$ & 0.033 & 1.08 & $-0.31(0.15)$ & 0.031 & 1.29 \\
\hline CANTAB AST $^{6}$ & & & & $0.70(0.18)$ & $<0.001$ & 1.05 & & & & $1.05(0.38)$ & 0.005 & 1.11 \\
\hline GCS $\times$ CANTAB AST & & & & $-0.050(0.015)$ & 0.001 & & & & & $-0.083(0.030)$ & 0.006 & \\
\hline CANTAB RTI $^{7}$ & & & & $0.46(0.17)$ & 0.006 & 1.14 & & & & & & \\
\hline GCS $\times$ CANTAB RTI & & & & $-0.034(0.014)$ & 0.013 & & & & & & & \\
\hline RAVLT-Delayed recall ${ }^{8}$ & & & & & & & & & & $0.31(0.14)$ & 0.023 & 1.21 \\
\hline Adjusted $\mathrm{R}^{2}$ & 0.083 & & & 0.095 & & & 0.020 & & & 0.095 & & \\
\hline
\end{tabular}

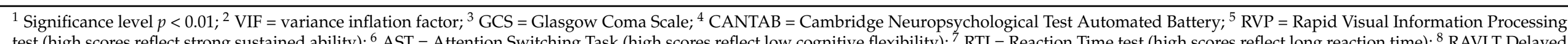

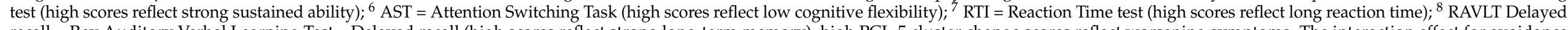

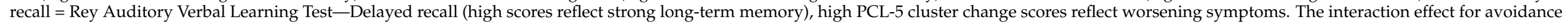

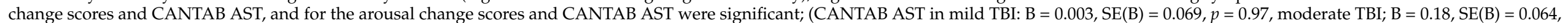

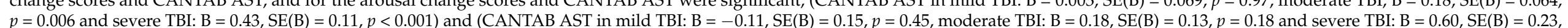

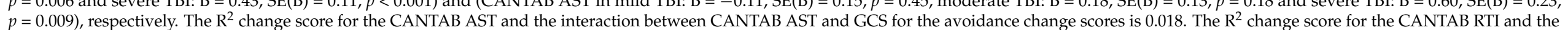

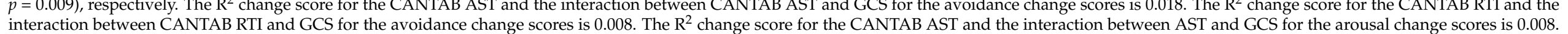


CANTAB AST performance was significantly related to avoidance change scores, but only in participants with moderate and severe TBI (see post-hoc test below Table 3, Figure S1). High AST scores (low cognitive flexibility) were related to high avoidance change scores, reflecting a worsening of avoidance symptoms. This also applied to RTI scores, but here, the interaction effect for the avoidance change score was not significant. High RTI scores (long reaction times) were related to high avoidance scores (worsening of avoidance symptoms).

CANTAB AST scores were significantly associated with arousal symptoms, but only in severe TBI (Figure S2). Higher AST scores (low cognitive flexibility) were related to deteriorating arousal symptoms. CANTAB RVP and RAVLT-Delayed recall scores were not significantly associated with arousal symptoms.

\section{Discussion}

Seeking to explore the effects of cognitive functioning six months post-TBI on the course of PTSD symptoms (as assessed with the PCL-5), we performed a complete case analysis on 671 participants with mild, moderate, or severe head injuries. Overall, nearly $25 \%(167 / 671)$ had PTSD symptom change scores of 10 or more points, with 87 participants having improved and 80 deteriorated. $11.8 \%$ had a probable diagnosis of PTSD at six months and showed significant improvement of their PTSD symptoms at 12 months compared to those without such a diagnosis, reflecting the difference in room for improvement. However, at the 12-month assessment, 32 new participants met criteria for delayed-onset PTSD.

Our hypothesis was only partially confirmed. We found a positive association between cognitive functioning and improvement of PTSD symptoms, however the strongest association was determined for sustained attention instead of verbal or visual memory, as hypothesized. Strong sustained attention (CANTAB RVP) was significantly related to improvement in PTSD symptoms after controlling for age, psychiatric history, baseline GCS and probable PTSD diagnosis. Looking at cognitive performance in relation to the four PTSD clusters, we found that none of the tests showed associations with the intrusion or cognition and mood clusters, while reaction time and cognitive flexibility correlated differentially with changes in the avoidance and arousal clusters.

\subsection{Course of PTSD Symptoms}

Since little research has been performed on the course of PTSD symptoms following TBI, comparisons with other findings are limited. We used a 10-point cut-off for the PCL5 change score to indicate clinically relevant changes. Weathers and colleagues (2013) suggested a threshold of 5-10 for a reliable and 10-20 points for a clinically significant change [25]. This is in line with the distribution of the change scores in our study sample. Alway and Gould et al. (2016) found that $17.6 \%$ of their cohort developed PTSD within the first four years after sustaining moderate or severe TBI, with a peak between six and 12 months [19]. About two-thirds were categorised as showing delayed-onset PTSD (at least 6 months post trauma), of which one in three had subsyndromal symptoms. Even though our sample also included participants with mild TBI, we found similar results: $16.6 \%$ had probable PTSD six months or one-year post-injury, and one in three of the participants suffering from delayed-onset PTSD (12 months post-TBI) showed subsyndromal symptoms at six months. As far as we are aware, the only two previous studies that investigated the course of post-TBI PTSD symptoms in relation to cognition either focused on children or veterans and did not describe the most relevant period between six and 12 months [16,18].

\subsection{Cognitive Functioning and the Course of PTSD Symptoms}

Our results show an inverse relationship between CANTAB RVP (high scores reflect better sustained attention) scores and PTSD change scores six- and 12-months post-injury (negative scores reflect improving symptoms), reflecting that strong sustained attention led to a reduction in PTSD symptoms. Comparing sustained attention three months post- 
injury with PTSD symptoms at six months in children, Guo et al. (2017) found the same association [18]. Additionally, having a probable PTSD diagnosis six months post-TBI predicts improvement of symptoms up to 12 months; this likely reflects the fact that they have more opportunity to recover compared to participants without PTSD. Nonetheless, in both groups with and without probable PTSD, cognitive performance at six months could predict either an improvement or a deterioration in PTSD symptoms. As mentioned above, the severity of TBI is another predictor of symptom change, with Alway and colleagues $[19,34]$ also reporting severe TBI to be associated with delayed-onset PTSD.

\subsection{Cognitive Functioning and the Course of PTSD Cluster Symptoms}

According to the DSM-5, the PTSD syndrome consists of four symptom clusters, of which the intrusion cluster is PTSD-specific. In the PCL-5, anxiety and depressive symptoms are captured in the cognition and mood, and arousal clusters, but they are not specific to PTSD [19,35]. Considering symptom changes in the four clusters, we found that none of the cognitive tests were significantly associated with change in the PTSD-specific intrusion cluster, which is similar to the findings by Guo et al. (2017) in a paediatric population [18]. They found a relationship between sustained attention scores at three months post injury and the non-PTSD-specific hyperarousal cluster at six months, which we could not replicate. In our sample, improvement in arousal symptoms was related to strong cognitive flexibility, but only in severe TBI. Additionally, we found that strong cognitive flexibility and reaction speed were related to an improvement in avoidance symptoms. Although this observation is a new finding for TBI, the relationship between cognitive flexibility and change in PTSD symptoms is a well-established association in populations with non-head trauma [36-38]. Ben-Zion et al. (2018) found that the cognitive training that improved cognitive flexibility also improved PTSD symptoms over time [36]. This suggests that interventions targeting cognitive flexibility positively affect PTSD development, support recovery, or a combination thereof, and may thus be a useful addition to traditional psychotherapeutic or pharmacological treatments [20].

\subsection{Limitations}

In the current study, we used the PCL-5 self-screening tool to monitor changes in PTSD severity but also to control for PTSD in our exploratory model. To arrive at this diagnosis of probable PTSD, we employed the symptom cluster method [24] but recognised that a formal diagnosis requires the structured assessment by a health professional. Further, the PCL-5 scores cannot be definitively related to the event that caused the TBI, as we have no pre-TBI data about PTSD symptoms. Additionally, change scores do not take the different symptom clusters into account and improvements can thus solely be attributable to changes in mood and not to recovery in PTSD-specific symptoms. To address this, we analysed changes for each of the four PTSD clusters separately. When interpreting cognitive test results, especially in patients with TBI, we need to acknowledge possible response bias due to lack of effort [39]. Although we did not include a formal performance validity test, examiners were instructed to record if low effort was apparent, and these test scores were removed from the database. Additionally, the TMT and RAVLT scores were examined and did not show evidence of systematic problems of low effort [40,41]. Further, to explore the impact of different cognitive variables on the course of PTSD, the choice of test is important. Great care was therefore taken in composing the test battery for the CENTER-TBI study; it needed to allow for cultural and language differences, since study participants were included from 19 European countries, and we needed to ensure that participants with mild, moderate and severe TBI would all be able to complete the full assessment. Still, both the TMT-B and CANTAB AST gauge cognitive flexibility, but we found only scores on the latter to be significantly associated with the observed changes. Of course, a cognitive test never addresses a single, isolated ability since cognitive functions are not separate constructs but overlap [42]. For example, cognitive flexibility also relies on attentional capacities and impaired sustained attention will affect learning 
and memory. Moreover, compared to the participants lost to follow-up, the participants in our study sample were younger, had received more education, were more often married or co-habiting and less frequently reported a history of substance abuse, while their TBI more often stemmed from road accidents, violent situations or a suicide attempt, whilst it more often stemmed from incidental falls in the lost-to-follow-up group. Furthermore, even though the cognitive covariates we computed were highly significant, we recognise that the adjusted $\mathrm{R}^{2} \mathrm{~s}$ were low, indicating that their predictive value was limited. We also recognise that model selection may increase the risk of type-I errors, and we therefore used a more stringent significance level of $p<0.01$. Finally, we did not control for the potential effects of psychopharmacological treatment or cognitive interventions, nor for life events that could have occurred after the TBI.

\section{Conclusions}

This study is the first to examine the course of self-reported PTSD symptoms (PCL-5) and associations with cognitive functioning in a relatively large civilian population with mild, moderate, and severe TBI. Overall, 13\% of the participants showed improvement and $12 \%$ deterioration in PTSD symptoms from 6 to 12 months post-injury. The participants with suspected PTSD six months post-TBI (11.8\%) showed an overall amelioration of PTSD symptoms compared to those that did not meet the PCL-5 criteria. Poor cognitive functioning has a negative impact on the course of PTSD symptoms following TBI. Assessment of reaction speed, sustained attention, and cognitive flexibility may accordingly help to identify patients at risk of developing (more severe) PTSD following TBI, with individual cognitive strengths and weaknesses informing treatment approaches. Future research should seek confirmation of our findings and, following recent reports [36,43], investigate whether cognitive training specifically aimed at enhancing reaction speed, sustained attention, and cognitive flexibility can alter the course of PTSD after TBI.

Supplementary Materials: The following are available online at https: / www.mdpi.com/article/ 10.3390/jcm10215109/s1, Table S1: Cognitive covariates, Table S2: Participant characteristics of the study sample and the participants with incomplete follow-up (FU) data, Table S3: Descriptive statistics for the PCL- 5 total scores at 6 and 12 months, differentiated for PTSD at 6 months, Table S4: Descriptive statistics for the PCL- 5 cluster change scores differentiated for PTSD at 6 months, Table S5: Linear Regression: Covariates Associated with PCL-5 Change Scores in Participants with a Probable PTSD Diagnosis $(n=79)$, Figure S1: Scatterplot of the PCL-5 avoidance change scores and the CANTAB AST differentiated for Glasgow Coma Scale, Figure S2: Scatterplot of the PCL-5 arousal change scores and the CANTAB AST differentiated for Glasgow Coma Scale.

Author Contributions: Conceptualization, D.L.G.V.P., A.I.R.M. and F.V.D.E.; methodology, D.L.G.V.P., A.I.R.M., F.V.D.E. and K.W.; software, D.L.G.V.P. and K.W.; validation, D.L.G.V.P., K.W. and L.W.; formal analysis, D.L.G.V.P.; investigation, D.L.G.V.P.; resources, A.I.R.M.; data curation, K.W., L.W. and A.I.R.M.; writing—original draft preparation, D.L.G.V.P.; writing—review and editing, A.I.R.M., K.W., L.W. and F.V.D.E.; visualization, D.L.G.V.P.; supervision, A.I.R.M. and F.V.D.E.; project administration, D.L.G.V.P.; funding acquisition, A.I.R.M. All authors have read and agreed to the published version of the manuscript.

Funding: The data used in the preparation of this manuscript were obtained in the context of CENTER-TBI, a large, collaborative research project supported by the European Union 7th Framework program (EC grant 602150). Additional funding was obtained from the Hannelore Kohl Stiftung (Germany), from OneMind (USA) and from Integra LifeSciences Corporation (USA). This research was additionally funded by the department of psychiatry of the Antwerp University Hospital.

Institutional Review Board Statement: The study was conducted according to the guidelines of the Declaration of Helsinki, and approved by the Institutional Review Board (or Ethics Committee) of each of the recruiting sites. More information can be found on: https / / www.center-tbi.eu/project/ ethical-approval (accessed on 30 July 2021).

Informed Consent Statement: Informed consent was obtained from all subjects involved in the study according to national and local regulations. 
Data Availability Statement: CENTER-TBI is committed to data sharing and in particular to responsible further use of the data. Hereto, we have a data sharing statement in place: https: / / www.center-tbi.eu/data/sharing/ (accessed on 30 July 2021). The CENTER-TBI dataset is hugely complex and the CENTER researchers wish to encourage correct and appropriate use of the data; this means that we encourage researchers to contact the CENTER-TBI team for any research plans and the Data Curation Team for any help in the appropriate use of the data, including the sharing of scripts. Requests for data access can be submitted online: http:/ / www.center-tbi.eu/data/ (accessed on 30 July 2021). The complete Manual for data access is also available online: https: / / www.center-tbi.eu/files/SOP-Manual-DAPR-20181101.pdf/ (accessed on 30 July 2021).

Conflicts of Interest: The authors declare no conflict of interest. The funders had no role in the design of the study; in the collection, analyses, or interpretation of data; in the writing of the manuscript, or in the decision to publish the results.

\section{References}

1. Cristofori, I.; Levin, H.S. Traumatic brain injury and cognition. Handb. Clin. Neurol. 2015, 128, 579-611. [CrossRef]

2. Rabinowitz, A.R.; Levin, H.S. Cognitive sequelae of traumatic brain injury. Psychiatr. Clin. N. Am. 2014, 37, 1-11. [CrossRef] [PubMed]

3. Wilson, L.; Stewart, W.; Dams-O'Connor, K.; Diaz-Arrastia, R.; Horton, L.; Menon, D.K.; Polinder, S. The chronic and evolving neurological consequences of traumatic brain injury. Lancet Neurol. 2017, 16, 813-825. [CrossRef]

4. Scholten, A.C.; Haagsma, J.A.; Cnossen, M.C.; Olff, M.; van Beeck, E.F.; Polinder, S. Prevalence of and risk factors for anxiety and depressive disorders after traumatic brain injury: A systematic review. J. Neurotrauma 2016, 33, 1969-1994. [CrossRef] [PubMed]

5. Van Praag, D.; Cnossen, M.C.; Polinder, S.; Wilson, L.; Maas, A. Post-Traumatic Stress Disorder after Civilian Traumatic Brain Injury: A Systematic Review and Meta-Analysis of Prevalence Rates. J. Neurotrauma 2019, 36, 3220-3232. [CrossRef]

6. Bryant, R. Post-traumatic stress disorder vs traumatic brain injury. Dialogues Clin. Neurosci. 2011, 13, 251-262. [CrossRef]

7. Howlett, J.R.; Stein, M.B. Post-Traumatic Stress Disorder: Relationship to Traumatic Brain Injury and Approach to Treatment. In Translational Research in Traumatic Brain Injury; Laskowitz, D., Grant, G., Eds.; CRC Press/Taylor and Francis Group: Boca Raton, FL, USA, 2016.

8. Merz, Z.C.; Roskos, P.T.; Gfeller, J.D.; Bucholz, R.D. Impact of psychiatric symptomatology on neuropsychological assessment performance in persons with TBI: A comparison of OEF/OIF veteran and civilian samples. Brain Inj. 2017, 31, 1422-1428. [CrossRef]

9. Scott, J.C.; Matt, G.E.; Wrocklage, K.M.; Crnich, C.; Jordan, J.; Southwick, S.M.; Krystal, J.H.; Schweinsburg, B.C. A quantitative meta-analysis of neurocognitive functioning in posttraumatic stress disorder. Psychol. Bull. 2015, 141, 105-140. [CrossRef]

10. Verfaellie, M.; Lafleche, G.; Spiro, A.; Bousquet, K. Neuropsychological outcomes in OEF/OIF veterans with self-report of blast exposure: Associations with mental health, but not MTBI. Neuropsychology 2014, 28, 337-346. [CrossRef]

11. Pineau, H.; Marchand, A.; Guay, S. Objective neuropsychological deficits in post-traumatic stress disorder and mild traumatic brain injury: What remains beyond symptom similarity? Behav. Sci. 2014, 4, 471-486. [CrossRef]

12. Shandera-Ochsner, A.L.; Berry, D.T.; Harp, J.P.; Edmundson, M.; Graue, L.O.; Roach, A.; High, W.M. Neuropsychological effects of self-reported deployment-related mild TBI and current PTSD in OIF/OEF veterans. Clin. Neuropsychol. 2013, 27, 881-907. [CrossRef]

13. Van Praag, D.; Van Den Eede, F.; Wouters, K.; Wilson, L.; Maas, A.I.R. Neurocognitive correlates of posttraumatic stress disorder following traumatic brain injury. J. Psychosom. Res. 2021, 145, 110448. [CrossRef]

14. Marx, B.P.; Doron-Lamarca, S.; Proctor, S.P.; Vasterling, J.J. The influence of pre-deployment neurocognitive functioning on post-deployment PTSD symptom outcomes among Iraq-deployed Army soldiers. J. Int. Neuropsychol. Soc. 2009, 15, 840-852. [CrossRef] [PubMed]

15. Parslow, R.A.; Jorm, A.F. Pretrauma and posttrauma neurocognitive functioning and PTSD symptoms in a community sample of young adults. Am. J. Psychiatry 2007, 164, 509-515. [CrossRef]

16. Vasterling, J.J.; Aslan, M.; Lee, L.O.; Proctor, S.P.; Ko, J.; Jacob, S.; Concato, J. Longitudinal associations among Posttraumatic stress disorder symptoms, traumatic brain injury and neurocognitive functioning in Army Soldiers deployed to the Iraq War. J. Int. Neuropsychol. Soc. 2018, 24, 311-323. [CrossRef]

17. Brewin, C.R.; Gregory, J.D.; Lipton, M.; Burgess, N. Intrusive images in psychological disorders: Characteristics, neural mechanisms, and treatment implications. Psychol. Rev. 2010, 117, 210-232. [CrossRef]

18. Guo, X.; Edmed, S.L.; Anderson, V.; Kenardy, J. Neurocognitive predictors of posttraumatic stress disorder symptoms in children 6 months after traumatic brain injury: A prospective study. Neuropsychology 2017, 31, 84-92. [CrossRef]

19. Alway, Y.; Gould, K.R.; McKay, A.; Johnston, L.; Ponsford, J. The Evolution of Post-Traumatic Stress Disorder following Moderate-to-Severe Traumatic Brain Injury. J. Neurotrauma 2016, 33, 825-831. [CrossRef] [PubMed]

20. Visser, E.; Gosens, T.; Den Oudsten, B.L.; De Vries, J. The course, prediction, and treatment of acute and posttraumatic stress in trauma patients: A systematic review. J. Trauma Acute Care Surg. 2017, 82, 1158-1183. [CrossRef] 
21. Maas, A.I.; Menon, D.K.; Steyerberg, E.W.; Citerio, G.; Lecky, F.; Manley, G.T.; Hill, S.; Legrand, V.; Sorgner, A.; CENTERTBI Participants and Investigators. Collaborative European NeuroTrauma Effectiveness Research in Traumatic Brain Injury (CENTER-TBI): A prospective longitudinal observational study. Neurosurgery 2015, 76, 67-80. [CrossRef]

22. Steyerberg, E.W.; Wiegers, E.; Sewalt, C.; Buki, A.; Citerio, G.; De Keyser, V.; Ercole, A.; Kunzmann, K.; Lanyon, L.; Lecky, F.; et al. Case-mix, care pathways, and outcomes in patients with traumatic brain injury in CENTER-TBI: A European prospective, multicenter, longitudinal, cohort study. Lancet Neurol. 2019, 18, 923-934. [CrossRef]

23. Wilson, J.T.; Pettigrew, L.E.; Teasdale, G.M. Structured interviews for the Glasgow Outcome Scale and the extended Glasgow Outcome Scale: Guidelines for their use. J. Neurotrauma 1998, 15, 573-585. [CrossRef] [PubMed]

24. Bagiella, E.; Novack, T.A.; Ansel, B.; Diaz-Arrastia, R.; Dikmen, S.; Hart, T.; Temkin, N. Measuring outcome in traumatic brain injury treatment trials: Recommendations from the traumatic brain injury clinical trials network. J. Head Trauma Rehabil. 2010, 25, 375-382. [CrossRef]

25. Weathers, F.L.; Litz, B.T.; Keane, T.M.; Palmieri, P.A.; Marx, B.P.; Schnurr, P.P. The PTSD Checklist for DSM-5 (PCL-5). 2013. Available online: https:/ / www.ptsd.va.gov (accessed on 1 July 2021).

26. Wortmann, J.H.; Jordan, A.H.; Weathers, F.W.; Resick, P.A.; Dondanville, K.A.; Hall-Clark, B.; Foa, E.B.; Young-McCaughan, S.; Yarvis, J.S.; Hembree, E.A.; et al. Psychometric analysis of the PTSD Checklist-5 (PCL-5) among treatment-seeking military service members. Psychol. Assess. 2016, 28, 1392-1403. [CrossRef]

27. Hoge, C.W.; Riviere, L.A.; Wilk, J.E.; Herrell, R.K.; Weathers, F.W. The prevalence of post-traumatic stress disorder (PTSD) in US combat soldiers: A head-to-head comparison of DSM-5 versus DSM-IV-TR symptom criteria with the PTSD checklist. Lancet Psychiatry 2014, 1, 269-277. [CrossRef]

28. Reitan, R.M. Trail Making Test: Manual for Administration and Scoring; Reitan Neuropsychology Laboratory: Tucson, AZ, USA, 1992.

29. Rey, A. L'examen psychologique dans les cas d'encéphalopathie traumatique. Arch. Psychol. 1941, 28, 21.

30. Schmidt, M. Rey Auditory Verbal Learning Test: A Handbook; Western Psychological Services: Los Angeles, CA, USA, 1996.

31. Cambridge Cognition. CANTAB Research Suite 6: Test Administration Guide; Cambridge Cognition Ltd.: Cambridge, UK, 2014.

32. IBM Corp. IBM SPSS Statistics for Windows, Version 25.0; IBM Corp: Armonk, NY, USA, 2017.

33. Teasdale, G.; Jennett, B. Assessment of coma and impaired consciousness. A practical scale. Lancet (London England) 1974, 2, 81-84. [CrossRef]

34. Alway, Y.; McKay, A.; Gould, K.R.; Johnston, L.; Ponsford, J. Factors associated with posttraumatic stress disorder following moderate to severe traumatic brain injury: A prospective study. Depress. Anxiety 2016, 33, 19-26. [CrossRef]

35. Nijdam, M.J.; Gersons, B.P.R.; Olff, M. The role of major depression in neurocognitive functioning in patients with posttraumatic stress disorder. Eur. J. Psychotraumatol. 2013, 4, 19979. [CrossRef]

36. Ben-Zion, Z.; Fine, N.B.; Keynan, N.J.; Admon, R.; Green, N.; Halevi, M.; Fonzo, G.A.; Achituv, M.; Merin, O.; Sharon, H.; et al. Cognitive Flexibility Predicts PTSD Symptoms: Observational and Interventional Studies. Front. Psychiatry 2018,9 , 477. [CrossRef]

37. Park, J.; Moghaddam, B. Impact of anxiety on prefrontal cortex encoding of cognitive flexibility. Neuroscience 2017, 345, 193-202. [CrossRef]

38. Shin, L.M.; Whalen, P.J.; Pitman, R.K.; Bush, G.; Macklin, M.L.; Lasko, N.B.; Orr, S.P.; McInerney, S.C.; Rauch, S.L. An fMRI study of anterior cingulate function in posttraumatic stress disorder. Biol. Psychiatry 2001, 50, 932-942. [CrossRef]

39. Wisdom, N.M.; Pastorek, N.J.; Miller, B.I.; Booth, J.E.; Romesser, J.M.; Linck, J.F.; Sim, A.H. PTSD and cognitive functioning: Importance of including performance validity testing. Clin. Neuropsychol. 2014, 28, 128-145. [CrossRef]

40. Boone, K.B.; Lu, P.; Wen, J. Comparison of various RAVLT scores in the detection of noncredible memory performance. Arch. Clin. Neuropsychol. 2015, 20, 301-319. [CrossRef]

41. Iverson, G.L.; Lange, R.T.; Green, P.; Franzen, M.D. Detecting exaggeration and malingering with the Trail Making Test. Clin. Neuropsychol. 2002, 16, 398-406. [CrossRef]

42. Sherman, E.M.S.; Brooks, B.L.; Iverson, G.L.; Slick, D.J.; Strauss, E. Reliability and Validity in Neuropsychology. In The Little Black Book of Neuropsychology; Schoenberg, M., Scott, J., Eds.; Springer: Boston, MA, USA, 2011. [CrossRef]

43. Jak, A.J.; Jurick, S.; Crocker, L.D.; Sanderson-Cimino, M.; Aupperle, R.; Rodgers, C.S.; Thomas, K.R.; Boyd, B.; Norman, S.B.; Lang, A.J.; et al. SMART-CPT for veterans with comorbid post-traumatic stress disorder and history of traumatic brain injury: A randomised controlled trial. J. Neurol. Neurosurg. Psychiatry 2019, 90, 333-341. [CrossRef] 